\title{
Translating Advances in Genomic Research into Clinical Practice: The Challenges Ahead
}

\author{
Seyed E. Hasnain ${ }^{a, c}$ Sharmistha Banerjee ${ }^{b}$ Nasreen Z. Ehtesham ${ }^{a}$ \\ anstitute of Life Sciences, Hyderabad University Campus, and ${ }^{b}$ Department of Biochemistry, University of

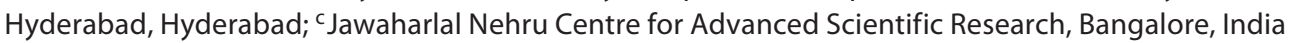

Ever since the discovery of the double helix model for DNA structure, there has been tremendous euphoria about the impact of DNA on human health. About 10 years ago, when the draft of the human genome sequence was announced, expectations about genomic medicine were further raised [1], despite the realization that the real impact on medicine and health care would happen as a function of decades and not in real time.

The Human Genome Project inspired several related collaborative efforts that supplemented cumulative knowledge on the genome-wide association of novel gene variants, gene modifications or alternate chromosomal loci with common diseases and disorder phenotypes. Careful examination of the available microarray data and identification and comparison of single nucleotide polymorphisms (SNPs) between patients and healthy populations have led to associations of many gene variants that so far were not suspected to be related to the phenotypes [2]. Basic understanding of human genes and proteins involved in the disease pathway and screening of mutations in critical enzyme(s) (mostly SNPs), and their association with disease predisposition [3], represent a significant advancement of genome research. The logarithmic growth of new 'omics' technologies, while quenching the thirst for knowledge of basic scientists who need unprejudiced
(C) 2011 S. Karger AG, Basel

1011-7571/11/0204-0392\$38.00/0

Fax +4161306 1234

E-Mail karger@karger.ch

www.karger.com
Accessible online at: www.karger.com/mpp evidence in support of the genetic elements of diseases, leaves clinicians at the other end of the translation highway with the difficulty of drawing meaningful, reproducible and categorical conclusions, and hence they are unable to use the data clinically. For research and technical advances, one always hopes that there will be actual application of the findings. Not surprisingly, therefore, the translation from 'bench to bedside' of genome-related basic research also faces tremendous challenges. Before really summarizing the challenges, one may have to stop and think if this research has the potential to be converted into clinical practice at all.

For multifactorial disorders like type 2 diabetes, obesity, polycystic ovary syndrome, autoimmune thyroid disease, hypertension or mood disorders, the record of genetic linkages and subsequent translation for disease prevention by adopting these into clinical practice has been far less productive through conventional routes of causal gene discovery. This was primarily because the magnitude of the effects of a single gene variation on susceptibility to these multifactorial diseases is too subtle to make any inferences and link a gene to a disease, which would have allowed detection by traditional single genesingle disease linkage approaches. This is where the $\mathrm{Hu}$ man Genome Project and related high-profile genomic 
efforts like genome-wide association analyses have had remarkable influence. The success of genetic screening and gene therapy in the control of disorders can be well exemplified by the tremendous reduction in the cases of $\beta$-thalassemia across e.g. Turkey, Greece, Cyprus, parts of the UK or Italy by well-executed strategies involving appropriate premarital counseling, carrier screening, prenatal diagnosis, selective termination of affected fetuses and, above all, long-reach public education $[4,5]$. In the last few years, the number of genes associated with type 2 diabetes increased from 3 to 20 [6]. Variation in a protein belonging to the family of zinc transporters (ZnT-8), called SLC30A8 (solute carrier family 30, member 8 ), that are expressed exclusively in pancreatic $\beta$-cells has been associated, thanks to genome-wide association analyses, with type 2 diabetes [7]. ZnT- 8 agonists have raised fresh therapeutic perspectives against type 2 diabetes.

In a few cases, advances in genomic research have also been translated into new therapies, specifically as a function of the genetic makeup of the patient, stage of disease and dose. Cancers, which are by and large less complex than other common disorders such as diabetes and hypertension, are some rare examples of the success of genomic medicine. The development of genomic-based therapeutics for cancer is often accompanied by a diagnostic test for the same. Three well-known examples of the commercial success of genomic medicine, resulting in the codevelopment of diagnostics and therapeutics approved by the US Food and Drug Administration, are Herceptin (trastuzumab) and the companion diagnostic immunohistochemistry test/PCR test for HER2 to treat breast cancer, Erbitux (cetuximab) and the companion epidermal growth factor receptor immunohistochemistry diagnostic test for treatment of colorectal cancer, and Gleevec (imatinib) along with the c-kit immunohistochemistry diagnostic for stomach cancer. However, the problem in many other cases has been the inability to accurately identify patients who will not respond to the drug, resulting in wrong clinical decisions.

The promise of personalized medicine by strategic advances as a consequence of the Human Genome Project is quietly reflected by certain pharmacogenetic and pharmacogenomic proceedings. Two such examples are the US Food and Drug Administration's permission for inclusion of genetic testing for rare skin reactions to carbamazepine primarily used for epilepsy and bipolar disorders [8] and the recent trial of HLA-B ${ }^{*} 5701$ screening of HIV patients for hypersensitivity to Abacavir, an antiHIV drug [9].

Translating Advances in Genomic Research into Clinical Practice
Encouraged by these desultory successes, genetic testing kits by several pharma companies have mushroomed over the past year. This is in parallel with an equal number of internet posts/blogs saying 'Genetic testing kits save your money - most don't work!' The clinical translations of genomic research are fundamentally challenged, apart from establishing analytical and clinical validity, by the clinical utility of such tests. The analytical validity of genomic variations with disease phenotype is largely established in the controlled environment of research laboratories, with the pharmaceutical industry attempting desperately to convert the proof of concepts into commercial kits, often ignoring that sample-handling errors are greater risks for these validations than errors in genotypic misclassification. A rigorous, transparent, nonmanipulative monitoring system is one of the main challenges that clinical translation of genomic research would have to face for proficiency of these platforms. Clinical validations would require bringing out these analytical corroborations from the controlled laboratory environment to actual clinical conditions to investigate sensitivity and specificity. The challenge here would be the accessibility of data across the world so that researchers can identify the geographic distribution or environmental linkages, if any, for these disorders.

Another challenge would be clinical validation of those genetic variations, which do not register high n-fold differences with healthy individuals. The genetic correlation of SNPs with disease predisposition has limited clinical utility simply because such SNPs do not reveal strongly influential haplotypes, and other haplotypes linked to predisposition to a disease together represent a very small fraction of heritable risk. Only discussions, data comparison and sharing amongst clinicians and researchers across the world can resolve these issues. Moreover, one must first establish the clinical efficacy of such tests and ask if these investigations would supplement the present disease management in any way. At the moment, this arena of clinical translation of genomic research is challenged by the relative deficit of well-performed clinical studies to indicate if normal and 'at-risk' populations would behave similarly to clinical interventions or protective lifestyle instructions in relation to the genetically linked multifactorial disorders. For instance, would it be correct to reassure a person who does not have any genetic trait of diabetes that he would never develop one?

The lack of awareness among patients and clinicians about these key determinants of success or failure of risk prediction has been further exacerbated by the fact that there is no single repository of publicly available informa-

Med Princ Pract 2011;20:392-394 
tion about the more than 2,000 genetic tests available in clinical laboratories or the large number of SNPs and the associated correlates of disease predisposition. The US Food and Drug Administration and the National Institutes of Health have stepped in, with support from the Department of Health and Human Services and other stakeholders, to set up a voluntary Genetic Testing Registry (http://www.ncbi.nlm.nih.gov/gtr) to provide usable access to such key information. Deaths due to infectious disease, which exceed mortality figures caused by all the wars in recorded history to date provide scanty information, if any, about genetic predisposition to an infectious pathogen. Therefore, it is yet to become a part of this registry. It is also important to note that once such a registry is fully established [10], the population that would benefit the most will come from the developed world where, paradoxically, the disease burden is far less than in developing and underdeveloped countries.

Genetic discriminations, socially and commercially, can be another big hurdle in the widespread application of genetic information [11] which can only be addressed by increasing genetic literacy among the general population.

Applying scientific discoveries to clinical practice has always occurred at a snail's pace, with an average of 17-20 years from laboratory to bedside application, and that too with only an average of $14 \%$ for scientific discoveries. It has been further pointed out that for the last 15 years, of the meager $5 \%$ of highly promising basic science discoveries approved for clinical use, only $1 \%$ could actually be practiced $[12,13]$. We now find this to be true [14] for genomics and genetic medicine, evident from the fact that except for small gains, as described above, in the form of gene-specific treatment for a few cancers, genetic risk assessment for drugs or disease and protective lifestyle interventions for some mendelian disorders, the societal impact of genomic medicine has been just like a drop in the ocean $[15,16]$.

While genomics and genetic medicine is becoming established, there is a need to accelerate the translational component by basic scientists, clinicians, drug companies, patients and the public at large working together; only then can real progress in personalized medicine be achieved. With newer and advanced technologies increasingly becoming available, the snail's pace of translating scientific discoveries into clinical practice should definitely improve. Time will tell whether 'genomics will widen or help heal the schism between research in medicine and public health' [17].

\section{Acknowledgement}

The senior author (S.E.H.) is a Robert Koch Fellow, Robert Koch Institute, Berlin, Germany, and a J.C. Bose National Fellow of the Department of Science and Technology, Government of India.

\section{References}

$\checkmark 1$ Varmus HE: Getting ready for gene-based medicine. N Engl J Med 2002;3:1526-1527.

2 Zhang Y, De S, Garner JR, Smith K, Wang S, Becker KG: Systematic analysis, comparison, and integration of disease based human genetic association data and mouse genetic phenotypic information. BMC Med Genomics 2010;3:1.

$\checkmark 3$ Kraft P, Hunter DJ: Genetic risk prediction - are we there yet? N Engl J Med 2009; 360:1701-1703.

4 Altay C, Yilgor E, Beksac S, Gurgey A: Premarital screening of hemoglobinopathies: a pilot study in Turkey. Hum Hered 1996;46: 112-114.

5 Angastiniotis MA, Hadjiminas M: Prevention of thalassaemia in Cyprus. Lancet 1981;317:369-371.

-6 Prokopenko I, McCarthy MI, Lindgren C: Type 2 diabetes: new genes, new understanding. Trends Genet 2008;24:613-621.

7 Sladek R, Rocheleau G, Rung J, Dina C, Shen L, Serre D, Boutin P, Vincent D, Belisle A,
Hadjadj S, Balkau B, Heude B, Charpentier G, Hudson T, Montpetit A, Pshezhetsky AV, Prentki M, Posner BI, Balding DJ, Meyre D, Polychronakos C, Froguel P: A genome-wide association study identifies novel risk loci for type 2 diabetes. Nature 2007;445:828-830.

8 FDA updates carbamazepine label to urge genetic testing for rare skin reactions (news item). Pharmocogenomics Reporter, December 19, 2007.

$\checkmark$ Mallal S, Phillips E, Carosi G, Molina JM, Workman C, Tomazic J, Jägel-Guedes E, Rugina S, Kozyrev O, Cid JF, Hay P, Nolan D, Hughes S, Hughes A, Ryan S, Fitch N, Thorborn D, Benbow A, PREDICT-1 Study Team: HLA-B*5701 screening for hypersensitivity to abacavir. N Engl J Med 2008;358:568-579. 10 Genetic Testing Registry. Bethesda, National Centre for Biotechnology, National Library of Medicine, 2010.

11 Ransohoff DF, Khoury MJ: Personal genomics: information can be harmful. Eur J Clin Invest 2010;40:64-68.
12 Khoury MJ, Gwinn M, Yoon PW, Dowling N, Moore CA, Bradley L: The continuum of translation research in genomic medicine: how can we accelerate the appropriate integration of human genome discoveries into health care and disease prevention? Genet Med 2007;9:665-674.

13 Contopoulos-Ioannidis DG, Alexiou G, Gouvias TC, Ioannidis JP: Medicine: life cycle of translational research for medical interventions. Science 2008;321:1298-1299.

14 Collins F: Has the gene revolution arrived? Nature 2010;464:674:675

15 Varmus HE: Ten years on the human genome and medicine. N Engl J Med 2010;362:20282029.

16 Feero W, Guttmacher AE, Collins FS: Genomic medicine - an updated primer. $\mathrm{N}$ Engl J Med 2010;362:2001-2011.

17 Khoury MJ, Gwinn M, Burke W, Bowen S, Zimmern R: Will genomics widen or help heal the schism between medicine and public health? Am J Prev Med 2007;33:310-317. 